\title{
Intervención preventiva por estudiantes de odontología a un paciente con parálisis cerebral. Descripción de un caso*
}

Preventive Care by Dental Students of an Patient with Cerebral Palsy. Case Report

Cuidado preventivo de estudantes de odontologia de um paciente com paralisia cerebral.

Relato de caso

Fecha de recepción: 17/06/2019 | Fecha de aceptación: 03/12/2019

Mónica LiLiana Cárdenas LaNCheros ${ }^{a}$

Fundación Universitaria San Martin. Bogotá, Colombia. lilica805@hotmail.com.

https://orcid.org/0000-0003-4093-218X

MARCELL OCAMPO ANJELLIS

Fundación Universitaria San Martin. Bogotá, Colombia. https://orcid.org/0000-0002-1608-

9797..anjelliso@gmail.com

\section{Isabel Cristina Mendoza González}

Fundación Universitaria San Martin. Bogotá, Colombia. isabel.mendoza@ sanmartin.edu.co.

https://orcid.org/0000-0002-9103-7789

EDITH DEL CARMEN HERnÁNDEZ RoJaS 
Fundación Universitaria San Martin. Bogotá, Colombia. edith.hernandez@sanmartin.edu.co. https://orcid.org/0000-0002-2874-068X

*Descripción de caso.

aCorrespondencia: lilica805@ hotmail.com

doi: https://doi.org/10.11144/Javeriana.uo38-81.ippe

Cómo citar: Cárdenas Lancheros ML, Ocampo Anjellis M, Mendoza González IC, Hernández Rojas EC. Intervención preventiva por estudiantes de odontología a un paciente con parálisis cerebral. Descripción de un caso. Univ Odontol. 2019 jul-dic; 38(81). https://doi.org/10.11144/Javeriana.uo38-81.ippe

\section{RESUMEN}

Antecedentes: En la ciudad de Bogotá, Colombia, la Fundación Fé efectúa una labor social con personas en condición de discapacidad. Allí, estudiantes de odontología de la Fundación Universitaria San Martín desarrollan actividades preventivas en salud oral. Objetivo: Describir una intervención preventiva por estudiantes de odontología a un paciente con parálisis cerebral (PC) y sus cuidadores. Descripción del caso: Se trató de una paciente institucionalizada con PC quien presentaba caries dental, enfermedad periodontal, maloclusión y sialorrea. Los estudiantes desarrollaron actividades preventivas y de manejo en salud bucal con los cuidadores de la paciente. 
Las actividades consistieron en instruirlos sobre cómo utilizar abridores de boca o tacos de mordida, cómo usar implementos de higiene bucal como crema fluorada, cepillo, seda y enhebradores y cómo aplicar enjuague bucal con gasas. Conclusiones: Esta actividad de instrucción y preventiva fue beneficiosa para los cuidadores al indicarles buenas prácticas de higiene oral para de la paciente. Asimismo, fue beneficiosa para la formación de los estudiantes de odontología con respecto al énfasis preventivo y educativo en poblaciones más necesitadas. Ellos ganaron en proactividad y conciencia sobre la labor social que pueden efectuar los profesionales con dichas poblaciones.

\section{Palabras clave}

discapacidad; educación en salud bucal; estudiante de odontología; odontología; odontología preventiva; práctica educativa; salud bucal

\section{ABSTRACT}

Background: In the city of Bogotá, Colombia, Foundation Fé carries out social work with people with disabilities. There, dental students from the San Martín University Foundation develop preventive activities in oral health. Objective: To describe a preventive intervention by dental students to a patient with cerebral palsy (CP) and their caregivers. Case description: It was an institutionalized patient with $\mathrm{CP}$ who had dental caries, periodontal disease, malocclusion, and sialorrhea. The students developed oral health preventive and care activities with the patients' caregivers. The activities consisted of instructing them on how to use mouth openers or bite plugs, how to use oral hygiene implements such as fluoridated toothpaste, toothbrush, dental floss, and 
threaders and how to apply mouthwash with gauze. Conclusions: This instructional and preventive activity was beneficial for caregivers by indicating good oral hygiene practices for the patient. It was also beneficial for the training of dental students with regard to preventive and educational emphasis on neediest populations. They gained in proactivity and awareness about the social work that professionals can carry out with these populations.

\section{Keywords}

dental student; dentistry; disability; educational practice; oral health; oral health education; preventive dentistry

\section{RESUMO}

Antecedentes: Na cidade de Bogotá, Colômbia, a Fundação Fé realiza trabalho social com pessoas com deficiência. Lá, estudantes de odontologia da Fundação Universitária San Martín desenvolvem atividades preventivas em saúde bucal. Objetivo: Descrever uma intervenção preventiva de estudantes de odontologia para um paciente com paralisia cerebral (PC) e seus cuidadores. Descrição do caso: Paciente institucionalizado com PC, com cárie dentária, doença periodontal, má oclusão e sialorréia. Os alunos desenvolveram atividades preventivas e cuidado em saúde bucal com os cuidadores da paciente. As atividades consistiram em instruí-los sobre como usar abridores de boca ou buchas, como usar instrumentos de higiene bucal, como creme fluoretado, pincel, seda e fios e como aplicar enxaguatório bucal com gaze. Conclusões: Essa atividade instrutiva e preventiva foi benéfica para os cuidadores, indicando boas práticas de higiene bucal para o paciente. Também foi benéfico para o treinamento de estudantes de 
odontologia no que diz respeito à ênfase preventiva e educacional nas populações mais necessitadas. Eles ganharam em proatividade e conscientização sobre o trabalho social que os profissionais podem realizar com essas populações.

\section{Palavras chave}

educação em saúde bucal; estudante de odontologia; incapacidade; odontologia; odontologia preventiva; prática educacional; saúde bucal

\section{INTRODUCCIÓN}

Según la organización Mundial de la Salud (OMS), el término Discapacidad incluye deficiencias, limitaciones de la actividad y restricciones de la participación, entendiendo por deficiencias los problemas que afectan a una estructura o función corporal, mientras que las limitaciones de la actividad son dificultades para ejecutar acciones o tareas y las restricciones de la participación se entienden como problemas para participar en situaciones vitales. Lo anterior entonces conlleva definir la discapacidad como un fenómeno complejo que refleja una interacción entre las características del organismo humano y las características de la sociedad en la que vive (1).

En la ciudad de Bogotá existen diversas instituciones cuya labor social es el manejo de personas con diferentes discapacidades, una de estas instituciones es la Fundación FE. Su misión es "Favorecer la inclusión social de la población en condición vulnerable, personas con discapacidad cognitiva y sus familias, brindando una atención integral basada en apoyos especializados de calidad en salud, educación y protección y orientada a elevar la calidad de vida, 
fortalecer vínculos familiares, movilizar la comunidad y consolidar redes de apoyo, en corresponsabilidad con el Estado y la familia (2).

La cobertura de la Fundación FE funciona mediante convenios o contratos con entidades públicas o privadas de todas las edades y estratos socio económico, recién nacido, niño, joven y adulto con discapacidad cognitiva, alteraciones en el desarrollo y limitaciones asociadas y familias con miembros en situación de discapacidad. Atiende en la Institución, el hogar del usuario y/o la comunidad, bajo la modalidad presencial ambulatoria o domiciliaria (2).

En este escenario, la facultad de Odontología de la Fundación Universitaria San Martín (FUSM) quiere resaltar el papel de la formación de sus estudiantes en la atención odontológica de promoción de la salud y prevención de la enfermedad al paciente diversamente funcional. Bajo estas prácticas pedagógicas, se les da la posibilidad de tener experiencias en un escenario real y multidisciplinar diferente de aquel donde ellos están acostumbrados a trabajar, lo que favorece a su formación como ciudadanos conscientes y actuantes, proporciona la inserción social de la institución a grupos vulnerables, además de que amplía su percepción del mercado de trabajo $(3,4)$. Es importante mencionar que los estudiantes deben conocer las condiciones discapacitantes de los usuarios a intervenir, con el fin de realizar una actividad acorde y que permita lograr el objetivo establecido por lo tanto esta va acompañada de una revisión de la literatura, que se basa en la búsqueda de artículos científicos de cada una de las discapacidades de los usuarios a intervenir y su respectiva discusión en mesa redonda, con la intención de que el alumno mejore su desempeño en la atención y sepa las características, diagnóstico, clasificación, características médicas de cada condición que puedan llegar a influir en un tratamiento odontológico, ya que los 
pacientes necesitan una atención especial y responsable. Tanto estudiantes como profesionales deben actuar con paciencia, comprensión, buena voluntad, saber los límites de a acuerdo a cada paciente y garantizar la mejor habilidad para realizar cualquier intervención (5). Al final de la rotación los estudiantes deben entregar un estudio de caso en donde queda plasmada la búsqueda de literatura científica actualizada sobre determinada condición y el relato de su intervención, lo que les permite: conocer con mayor alcance las deficiencias (neurológicas, psíquicas, motrices, síndromes y otros) siendo conducidos a trabajar en equipo, desarrollar autonomía intelectual, responsabilidad individual y colectiva, adquirir espontaneidad para la discusión, diferentes formas de argumentación y comunicación, estimular competencias afectivas, articulación teórico práctica, búsqueda y uso de información relevante $(6,7)$ y permitiendo la autonomía del estudiante en la gestión de su conocimiento y aportando herramientas que lo pueden auxiliar en su formación integral y humana como odontólogo egresado de la Institución y capacitados en los diferentes niveles de atención a la salud, todo lo anterior con un fin mayor que es el de promover calidad de vida a estos pacientes $(8,9)$.

El objetivo de este artículo fue describir una práctica pedagógica con estudiantes de pregrado de odontología de X semestre, que cursaron el área temática de Ciencias Básicas, Medicina Oral Y Cirugía del programa de Odontología en la Fundación Universitaria San Martín, durante la rotación dirigida a la sensibilización del estudiante frente a la atención preventiva de un paciente en condición de discapacidad, la búsqueda de literatura científica y su aplicación reflexiva en la intervención individualizada tomado como ejemplo a una paciente con parálisis cerebral de tipo hipotónico, todo esto con el fin del afianzar e integrar sus conocimientos, permitiendo que el futuro 
odontólogo sea entrenado para disminuir las barreras de atención en salud al paciente discapacitado y a dar una adecuada orientación preventiva de las enfermedades bucales a sus cuidadores.

\section{REVISIÓN DE LA LITERATURA}

\section{Discapacidad}

La Clasificación Internacional del Funcionamiento, de la Discapacidad y de la Salud (CIF) (10), define la discapacidad como: "Un término genérico que engloba deficiencias, limitaciones de actividad y restricciones para la participación y denota los aspectos negativos de la interacción entre personas con un problema de salud (como parálisis cerebral, síndrome de Down o depresión) y factores personales y ambientales (como actitudes negativas, transporte al discapacitado y edificios públicos inaccesibles para las diferentes condiciones de discapacidad, así como la falta de apoyo social)".

Según el Departamento de Estadística DANE, Colombia no tiene una cifra exacta de las personas con discapacidad, no obstante el Censo del DANE de 2005 captó a 2.624 .898 (6,1 \%) personas que refirieron tener alguna discapacidad (11) y actualmente gracias al Registro para la Localización y Caracterización de Personas con Discapacidad en Colombia (RLCPD) se pueden recolectar e informar datos actualizados de las personas con discapacidad desde el año 2002 disponiendo de información a nivel nacional, para llevar a cabo los programas y proyectos dirigidos a garantizar los derechos de las personas con discapacidad en Colombia. A corte de 30 de septiembre de 2019 hay un total de 1'521.114 personas registradas (12). 
Según la Sala situacional de las Personas con Discapacidad (PCD) del Ministerio de Salud y Protección Social, Oficina de Promoción Social, en Colombia en el 2018 el 58 \% (796.169) de las personas con discapacidad registradas en el RLCPD son mayores a 50 años de edad, el $12 \%$ (167.769) son menores de edad y el $51 \%$ (691.149) son mujeres. Por cada 100.000 habitantes hay 2.723 personas con discapacidad y el $80 \%$ de ellas refirieron pertenecer a los estratos socioeconómicos uno y dos. Por grupos de edad el $47 \%(46,409)$ de los niños menores de 15 años y las alteraciones que más les afecta es el sistema nervioso, seguido por el movimiento del cuerpo $20 \%(20,508) .(13)$

\section{Parálisis cerebral}

Como sostienen Giraldo et al. (14) Una de las discapacidades más frecuentes descritas en la literatura es la parálisis cerebral, adicionalmente el Comité Ejecutivo Internacional para la Definición de Parálisis Cerebral, propuso definirlo como "un grupo de trastornos permanentes del desarrollo del movimiento y postura, que causa limitación de la actividad, que se atribuyen a las perturbaciones no progresivas que ocurrieron en el cerebro fetal o infantil en desarrollo. Los trastornos motores de la parálisis cerebral suelen ir acompañados de alteraciones de sensación, percepción, cognición, comunicación, comportamiento, problemas de tipo músculo esqueléticos, condiciones secundarias como la epilepsia (15)

De otro lado, Jan, et al (16) dicen que los pacientes con parálisis cerebral también padecen de alteraciones nutricionales y mayor riesgo de problemas bucales conllevando a una morbilidad importante que puede comprometer su bienestar e impactar negativamente en su calidad de vida, 
es por esto que las visitas al odontólogo deben formar parte de la evaluación inicial de cualquier niño con parálisis cerebral.

Así mismo, la OMS $(17,18)$ afirma que la parálisis cerebral no tiene cura y posee pocas intervenciones modificadoras de la enfermedad lo que convierte al manejo de los síntomas en el pilar del tratamiento. Para garantizar que las intervenciones tengan resultados significativos para las personas con parálisis cerebral, estas deben relacionarse con el marco de la Clasificación Internacional de Funcionamiento, Discapacidad y Salud para la discapacidad: estructura corporal, actividad y participación.

La parálisis cerebral (PC) es la causa más común de discapacidades motoras pediátricas, que afectan aproximadamente 2.4 por 1000 nacidos vivos (19) con una etiología variada, lo que hace que el estudio y entendimiento de sus factores asociados (tabla 1) se puedan prevenir, detectar precozmente y en consecuencia darles un correcto acompañamiento (20). Según el registro para la localización y caracterización de las personas con discapacidad según su estructura o función corporal afectada en este caso el movimiento del cuerpo, manos y piernas al 2010 se encontraban registradas 55.796 personas en Bogotá (12).

TABLA 1

FACTORES De Riesgo De La PARÁlisis CEREBral. ModifiCAdo DE PÓo P., 2008. PARÁlisis CEREBRAL INFANTIL. Protocolos Diagnóstico Terapeúticos de la AEP (Asociación EsPañola de Pediatría): Neurología PEDIÁTRICA: WWW.AEPED.ES/PROTOCOLOS/

\begin{tabular}{ll}
\hline \multicolumn{1}{c}{ Factor } & Descripción \\
\hline Factores prenatales & Factores maternos: \\
\hline
\end{tabular}


- Alteraciones la coagulación, enfermedades autoinmunes, HTA, infección intrauterina, traumatismo, sustancias tóxicas, disfunción tiroidea

- $\quad$ Alteraciones de la placenta

- Trombosis en el lado materno, trombosis en el lado fetal, cambios vasculares crónicos, infección.

Factores fetales:

- Gestación múltiple

- Retraso crecimiento intrauterino

- Polihidramnios, hidrops fetalis, malformaciones

Factores perinatales

Factores postnatales
Prematuridad, bajo peso Fiebre materna durante el parto, Infección SNC o sistémica Hipoglucemia mantenida, hiperbilirrubinemia Hemorragia intracraneal Encefalopatía hipóxico-isquémica Traumatismo, cirugía cardíaca, ECMO
Infecciones (meningitis, encefalitis) Traumatismo craneal Estatus convulsivo Parada cardio-respiratoria Intoxicación Deshidratación grave

De otro lado, Sanger et al. (21) afirmaron que la complejidad de la parálisis cerebral es clara a partir de sus diversas clasificaciones ya que la parálisis cerebral se puede describir de acuerdo con el sitio anatómico de la lesión cerebral (corteza cerebral, tracto piramidal, sistema extrapiramidal o cerebelo); síntomas y signos clínicos (espasticidad, discinesia -distónico y formas de coreatosiso ataxia); topografía o afectación de extremidades (diplegia, cuadriplejía o hemiplejia); momento de presunto daño (prenatal, natal o posnatal) y clasificación de grado del tono muscular (isotónico, hipotónico o hipertónico) (figura 1 y 2). La mayoría de los pacientes se encuentran clasificados dentro de la categoría espástica, siendo ésta abarcada hasta en un 75 a $80 \%$, mientras que el resto se divide en los otros tipos y en los diferentes trastornos espásticos se da la clasificación topográfica, así en el caso de la Hemiplejía sólo un hemicuerpo comprometido con mayor afección en miembro superior que inferior, mientras que en la diplejía se observa un mayor retraso en el desarrollo psicomotor, especialmente en el área motora, se presenta espasticidad con contractura de músculos aductores y flexores de la cadera. La cuadriplejía es la más grave de todas, pues observa compromiso de las cuatro extremidades, pero siempre acompañada de una hipotonía del 
tronco e hipertonía apendicular, no logra el control cefálico y por lo tanto del tronco. En cuanto a la paraplejia se refiere a la afectación de los miembros inferiores, en la monoplejia se encuentra afectado un único miembro, superior o inferior mientras que en la triplejia se ven tres miembros afectados $(22,23)$.

\section{FIGURA 1}

FORMAS CLÁSICAS DE CLASIFICAR LA PARÁLISIS CEREBRAL. COPYRIGHT 2019 POR EFISIOPEDIATRIC. 2016 [CITED 11 OCTOBER 2019]. AVAILABLE FROM: HTTPS://EFISIOPEDIATRIC.INFO/WP-
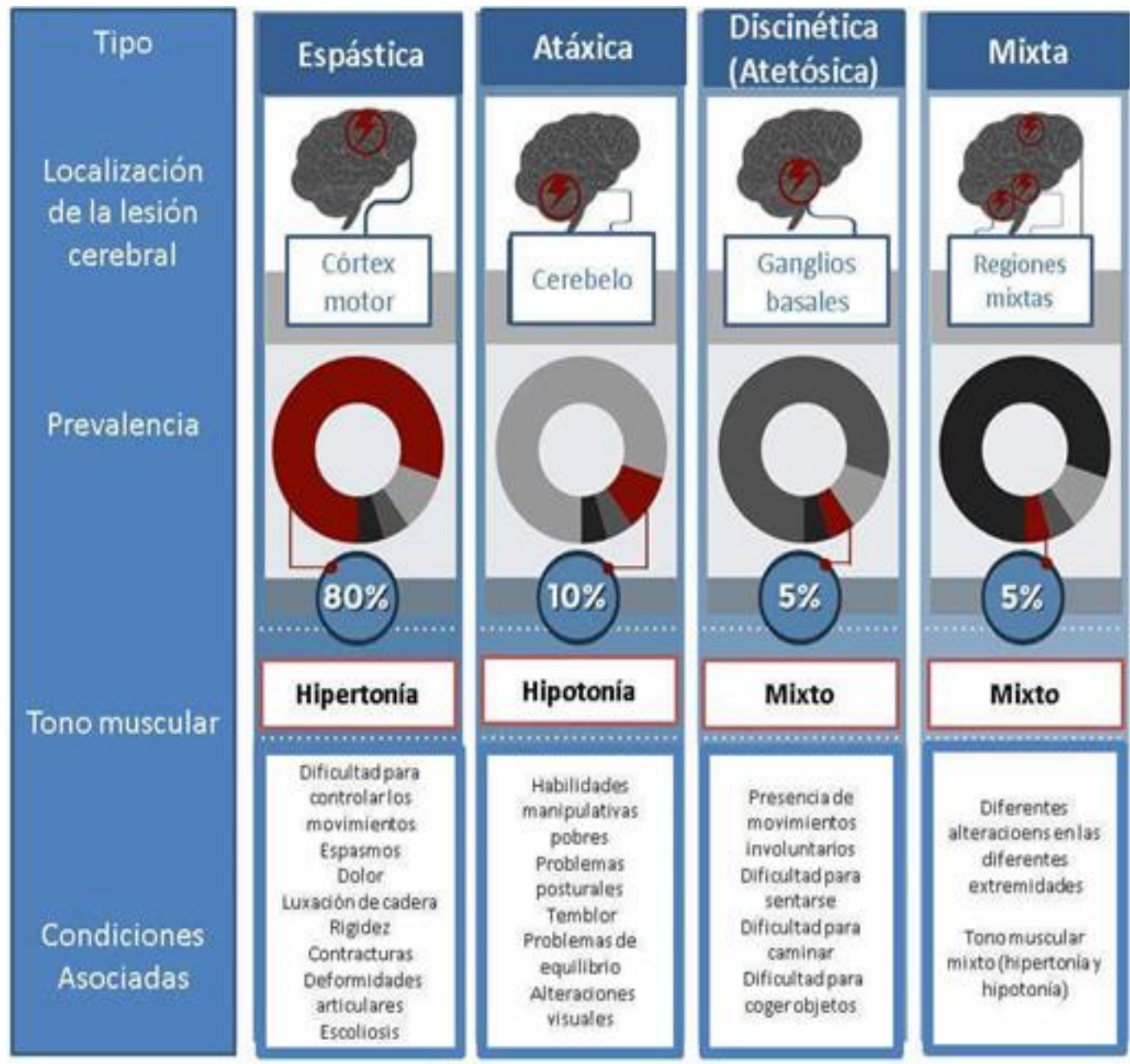
TIPOS DE PARÁLISIS CEREBRAL SEGÚN LAS ÁREAS DEL CUERPO AFECTADAS. COPYRIGHT 2019 POR EFISIOPEDIATRIC. AFECTADAS. 2016 [CITED 11 OCTOBER 2019]. AVAILABLE FROM: HTTPS://EFISIOPEDIATRIC.INFO/WPCONTENT/UPLOADS/2016/10/CUADRO-TIPOS.JPG
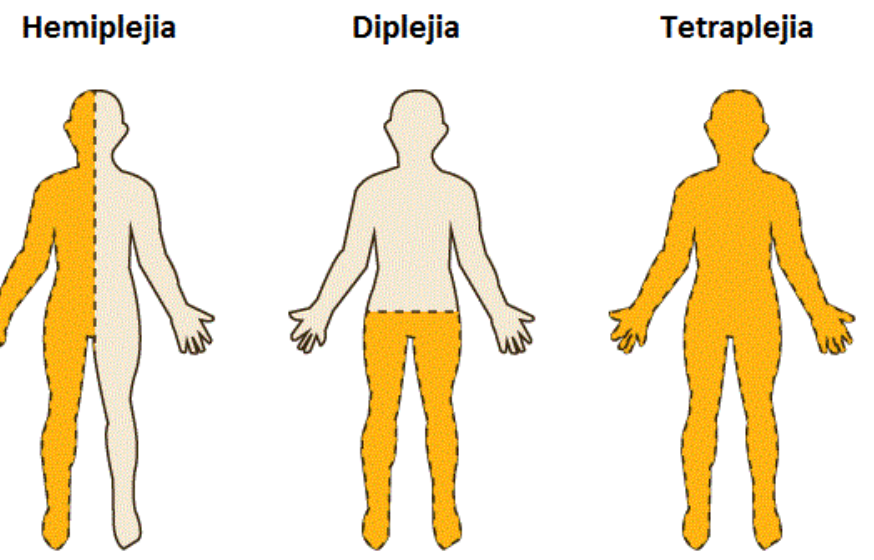

Los desórdenes del movimiento depende del daño cerebral pueden presentar atetosis, que son movimientos involuntarios lentos, la corea que se manifiesta en movimientos abruptos, torpes e irregulares; coreoatetósicos son una combinación de ambos; la distonía que son los movimientos lentos, rítmicos, con cambio en el tono y por último la ataxia que es la inestabilidad con movimientos incoordinados, asociados a nistagmus, disartria y marcha con base de sustentación amplia (24).

Con relación al tono muscular se habla tanto de pacientes hipertónicos (tono incrementado) y pacientes hipotónicos (resistencia disminuida al estiramiento pasivo), la hipotonía es poco persistente en la parálisis cerebral y se acompaña de hiperreflexia osteotendinosa, que persiste más allá de los 2-3 años y a menudo se transforma en ataxia o espasticidad (25). 
De otro lado, la epilepsia es más frecuente en niños y adultos con parálisis cerebral y los que padecen formas más severas de parálisis cerebral como por ejemplo la unilateral espástica, es común que se presente la epilepsia focal, en pacientes con afectación bilateral severa, hasta $50 \%$ tienen epilepsia generalizada para lo cual el control de las convulsiones puede ser difícil de lograr, además, las dificultades diagnósticas también pueden surgir si el trastorno del movimiento subyacente imita los episodios epilépticos (26). El $43 \%$ de niños con parálisis cerebral (rango 35 $\%$-62 \%) desarrollan convulsiones, generalmente en el primer año de vida requiriendo a menudo múltiples fármacos antiepilépticos para mejorar la calidad de vida y sus riesgos asociados, los efectos cognitivos y del comportamiento son el problema principal con la medicación antiepiléptica y evitando la politerapia, la titulación lenta y el uso de la dosis efectiva más baja pueden minimizarlos $(27,28)$.

Todos los tipos de ataques epilépticos se observan en niños con parálisis cerebral y en general, las convulsiones de inicio focal (a menudo con generalización secundaria) predominan en niños con espástica unilateral (hemipléjica) o parálisis cerebral espástica bilateral (diplejica) mientras que la epilepsia en otros subtipos motores de parálisis cerebral se caracteriza por múltiples tipos de convulsiones generalizadas en el mismo paciente, estas incluyen tónico-clónico generalizado, tónico, atónico, ausencia y ataques atípicos de ausencia; los espasmos infantiles ocurren en algunos bebés, particularmente en aquellos con microcefalia y parálisis cerebral de carácter tetrapléjico o atónico espástico (29). 


\section{Salud bucal en niños con parálisis cerebral}

Personas con discapacidades, tanto físicas como intelectuales requieren de más atención y supervisión en las actividades cotidianas incluidas las de salud bucal. Proporcionar una respuesta eficiente a esta demanda requiere una capacitación adecuada para profesionales en un entorno clínico sin barreras físicas, lo que permite un fácil acceso a esta población y que puedan recibir tratamiento de acuerdo con sus necesidades (30).

Los niños con parálisis cerebral tienen un mayor riesgo de desarrollar problemas dentales esto puede crear una morbilidad significativa que puede afectar su bienestar e impactar negativamente su calidad de vida (31). Por lo cual el acompañamiento de profesionales del área de la salud bucal debe ser una prioridad. Se ha demostrado que dependiendo de la gravedad de la afección neurológica, el riesgo de enfermedades en la cavidad bucal es mayor como resultado de las dificultades motoras y la coordinación que se presenta en la parálisis cerebral, así como los limitados cuidados en higiene oral (16), a continuación en la Tabla 2 se resumen algunos factores predisponentes a las enfermedades bucales en los niños que presentan parálisis cerebral.

TABLA 2

FACTORES QUE POSIBLEMENTE PREDISPONEN A LA ENFERMEDAD DENTAL EN NIÑOS CON PARÁLISIS CEREBRAL. ModifiCAdo DE JAN, B. M., \& JAN, M. M. (2016) DENTAL HEALTH OF CHILDREN WITH CEREBRAL PALSY.

NEUROSCIENCES, 21(4): 314-318. HTTP://DOI.ORG/10.17712/NSJ.2016.4.20150729 


\begin{tabular}{ll}
\hline Debilidad motora o incoordinación & $\begin{array}{l}\text { Incapacidad para mantener la higiene oral } \\
\text { Dependiendo de un cuidador para el riesgo de cuidado personal de } \\
\text { trauma dental } \\
\text { Incapacidad para mantener la higiene oral }\end{array}$ \\
Retraso mental & Dependencia de un cuidador para el cuidado personal \\
Parálisis pseudobulbar & $\begin{array}{l}\text { Dificultades en la masticación y deglución } \\
\text { Riesgo de caries y erosiones dentales } \\
\text { Exceso de babeo (sialorrea) }\end{array}$ \\
La enfermedad por reflujo gastroesofágico & $\begin{array}{l}\text { Regurgitación y vómitos recurrentes causando erosiones dentales } \\
\text { Desnutrición }\end{array}$ \\
& $\begin{array}{l}\text { Poca ingesta de calcio } \\
\text { Deficiencia de vitamina D }\end{array}$ \\
\hline
\end{tabular}

\section{DESCRIPCIÓN DEL CASO}

Se trató de una niña de 6 años de edad, quien asiste a la Fundación FE, con antecedentes de Parálisis cerebral tipo hipotónico (figura 1), epilepsia focal controlada. La paciente se encuentra en un programa de protección del Instituto Colombiano de Bienestar Familiar ICBF), para la restitución de algún derecho fundamental vulnerado, en un salón junto con otros 6 niños, con un diagnóstico común que es la parálisis cerebral, todos los niños tiene sus miembros tanto superiores como inferiores afectados (figura 2). Ellos están a cargo de una profesional en Fisioterapia que los recibe en las mañanas, les administra los medicamentos, los alimenta, les cambia el pañal. Es importante aclarar que la paciente por hacer parte de un grupo de niños protegidos por el ICBF, el manejo de sus datos es reservado.

El primer día de rotación los estudiantes reconocieron la paciente asignada, como primera experiencia de atención a un paciente con P.C y para tomar registro de la valoración inicial, se realizó una adaptación del lugar para poder recolectar la información teniendo en cuenta la 
discapacidad y el diagnóstico de la paciente, guiados por el docente tutor y la profesora de la institución, para lo cual se ubicó a la paciente en una camilla ubicada en el mismo salón, se procedió a adaptar a la paciente a la presencia de los estudiantes, usando los toma de signos vitales como apoyo. Se observó a nivel muscular relajación extrema (hipotonicidad), la obtención de dicha información se facilitó ya que la paciente se encontraba tranquila y colaboradora.

En una segunda sesión se realizó una mesa redonda donde se discutió reflexivamente la condición médica de la paciente (que es la revisión temática descrita en párrafos anteriores) y ya teniendo un conocimiento más claro se procedió a realizar un manejo preventivo de salud bucal, un examen odontológico exigido por el ICBF.

\section{Materiales usados}

Se llevó el instrumental básico (espejo bucal, pinza algodonera, sonda), líquido revelador de placa, seda dental (porta-sedas), babero, abridores de boca, bajalenguas, cepillo de cerdas suaves, crema dental con flúor (1100 ppm de flúor), enjuague bucal (clorhexidina 0.12), toallas absorbentes y gasas.

\section{Descripción de la práctica}

El cuerpo de la paciente quedó inclinado hacia adelante con las almohadas y la cabeza girada hacia un lado, para disminuir el riesgo de atragantarse y facilitar la remoción de saliva y crema dental en el momento del cepillado, ya que la paciente presenta dificultades en el movimiento normal de la musculatura perioral, dificultándose escupir (figura 3) También se sugiere cepillar a la paciente en su silla de ruedas, ya que ésta tiene la posibilidad de inclinar el espaldar hacia adelante (figura 4). 
INSTRUCCIONES DE POSICIÓN DEL PACIENTE PARA EL CEPILLADO

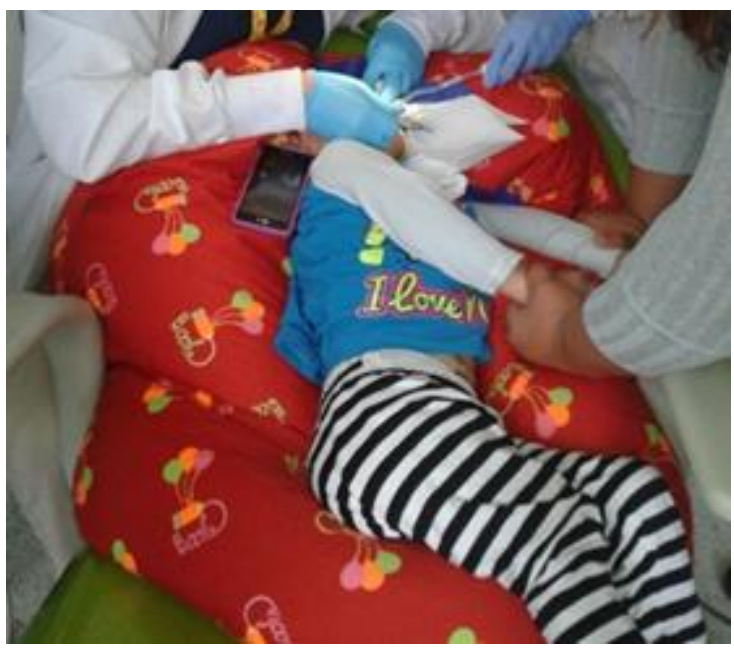

FIGURA 4

POSICIÓN PARA EL CEPILLADO EN SILLA DE RUEDAS

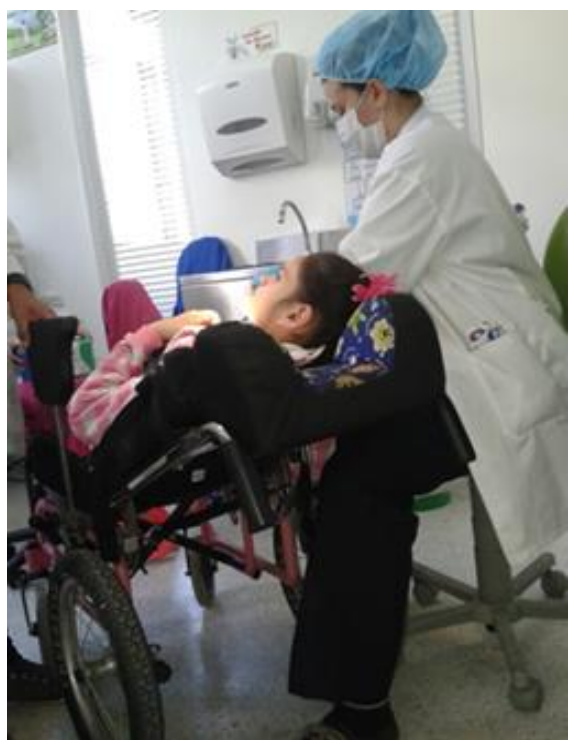


Se aplicó líquido revelador de placa con el fin de que la profesora del salón y los estudiantes observarán en qué lugares se debería mejorar la higiene bucal de la paciente, se toma una gasa y se impregna de líquido revelador, y se pasa con la gasa por todas las superficies dentales. Se realizó el índice de O’Leary para determinar el porcentaje de placa bacteriana blanda en cada superficie dental. Se sugiere el uso de líquido revelador de placa a los cuidadores para comprobar la efectividad del cepillado

Se hizo una demostración de cómo hacer correctamente la higiene bucal de la paciente, a los estudiantes y a la profesora encargada del salón, Se sugiere una posición del cuidador que permita visualizar todas las superficies de los dientes, detrás del paciente y un poco lateralizado. Esta posición puede variar procurando una mejor comodidad y accesibilidad a la boca.

Se indicó la cantidad de crema a usar en poca cantidad dependiendo si la paciente ingiere la crema o no, con una concentración mínima de 1000 ppm de flúor y en cuanto a la cantidad, se sugiere el tamaño similar a un grano de arveja. Para población pediátrica se sugiere la marca Colgate Kids o la Oral B Kids, como la paciente no puede escupir recurrió a toallas absorbentes y gasas.

Se utilizaron los dedos como pinzas, permitiendo separar los labios con delicadeza y mejorar la visibilidad

La técnica de cepillado es variada, se sugiere para pacientes pediátricos un cepillo de cabeza pequeña, cerdas muy juntas y suaves, el mango grueso y largo, cepillar mínimo dos veces al día. en casos donde el paciente es más independiente del cuidador se sugiere adaptar los mangos de los 
cepillos y estimular la inclusión del paciente en sus prácticas de higiene oral. El sentido del cepillado desde la encía hacia la superficie coronal de los dientes y en las superficies de masticación en forma circular, tanto en dientes anteriores como posteriores se debe prolongar el tiempo de cepillado hasta 3 minutos y finaliza con un barrido suave de la superficie dorsal de la lengua Se hizo una aclaración, la cual es que en el caso del paciente con parálisis cerebral espástica, existe la dificultad de que el paciente abra la boca y permita el cepillado correctamente, para lo cual se le sugierió a la profesora y a los estudiantes rotantes dos elementos de apoyo que son el abridor de boca o crear un abrebocas individualizados utilizando bajalenguas (figura 5), el número de bajalenguas varía dependiendo del tamaño de la boca y la apertura del paciente, se unen con esparadrapo y se usa en una hemiarcada para cepillar el lado contralateral. Así mismo, se indicó la importancia de tener en cuenta que para los niños mayores que no desean o no pueden cooperar físicamente, el odontólogo debe enseñar a los padres las técnicas correctas de cepillado y las formas de restringir o contener los movimientos del niño cuando sea necesario; Así en un primer escenario el niño se coloca en el regazo de los padres para estabilizar la cabeza con una mano mientras usa la otra para cepillarle los dientes; en un segundo caso con un niño mayor, puede recostársele en una silla o en la cama y el padre inclina la cabeza hacia atrás con una mano mientras los dientes se cepillan. 

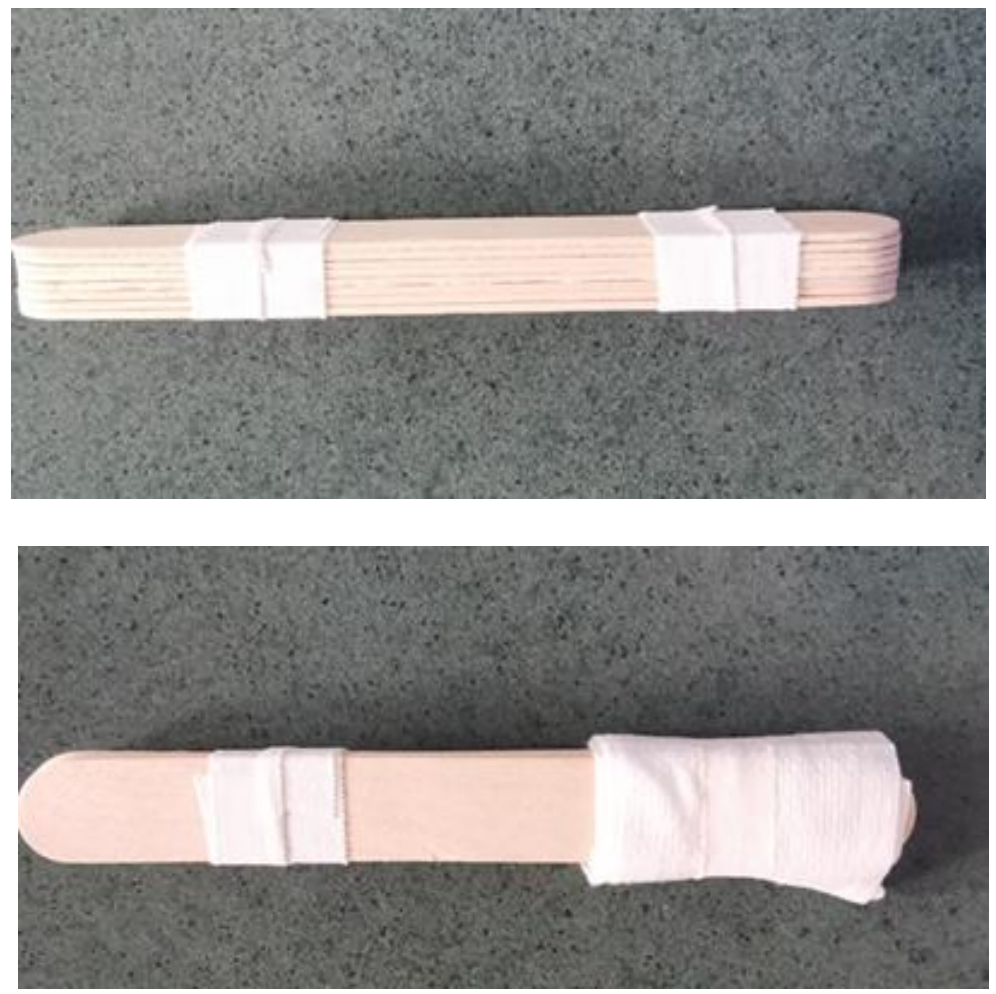

Se enfatizó en el uso de la seda dental y como la paciente se encontraba muy colaboradora y tranquila se usó la técnica tradicional del uso de seda dental, cortando aproximadamente $30 \mathrm{~cm}$ de hilo, enrollándola una punta en el dedo índice de la mano izquierda y el resto el dedo índice de la mano derecha dejando de 6 a $8 \mathrm{~cm}$ libres, se inserta la seda dental suavemente que no impacte la papila dental, se enrolla la seda usada y se desenrolla la seda limpia, repitiendo la maniobra en todos los espacios interdentales. Se aclara que en caso de que se presente dificultad en el uso de la seda porque algunos pacientes con parálisis cerebral tienen limitaciones en la apertura se sugiere otra herramienta que es el porta seda. 
Se implementó el uso de enjuague bucal, dándose a conocer una forma alternativa de su uso, para pacientes que no pueden enjuagarse, utilizando una gasa empapada y escurrida en clorhexidina al $0,05 \%$ y enrollada en el dedo índice, pasándola por todas las superficies dentales y gingivales, con el fin de controlar la formación de placa bacteriana, pues común encontrar dificultades en los pacientes con discapacidad en el control mecánico adecuado de la placa.

\section{Hallazgos en la paciente y manejo dado por los estudiantes}

Hábitos nutricionales: la paciente ingiere sus alimentos licuados (dieta líquida completa) 5 veces al día, la profesora encargada de su cuidado los suministra con cuchara vía oral, debido a la hipotonicidad de la paciente y su incapacidad de masticar.

Hábitos de higiene oral: la paciente es cepillada en la fundación cuando le queda tiempo a la profesora encargada del salón, con crema dental y cepillo, no usan la seda dental, ni enjuagues. la profesora afirma que muchas veces no le queda tiempo para realizar esta práctica pues debe estar a cargo prácticamente sola, de los 6 niños que dependen completamente de ella.

Examen clínico intraoral: se evidenció en la paciente Maxilares ojivales, Lengua y musculatura perioral hipotónica, Sialorrea, porcentaje alto placa bacteriana $(100 \%$, todas las superficies dentales fueron teñidas) y encías inflamadas asociado a la presencia de placa bacteriana, presencia de placa calcificada en los incisivos inferiores, presencia de dentición mixta de la primera transición (tabla 3) y procesos cariosos activos. 
TABLA 3

Examen Dental de la Paciente de estudio

\begin{tabular}{llll}
\hline & Primer cuadrante & & Segundo cuadrante \\
\hline 55 & Sellante & 65 & Sellante \\
\hline 54 & Sellante & 64 & Sellante \\
\hline 53 & Sano & 63 & Sano \\
\hline 52 & Sano & 62 & Sano \\
\hline 11 & En erupción & 61 & Exfoliado \\
\hline & Cuarto cuadrante & & Tercer cuadrante \\
\hline 85 & Caries extensa & 75 & Cavidad detectable \\
\hline 84 & Caries extensa & 74 & Sano \\
\hline 83 & Sano & 73 & Sano \\
\hline 42 & En erupción & 32 & En erupción \\
\hline 41 & En erupción & 31 & En erupción \\
\hline
\end{tabular}

Por último, se realiza un examen clínico odontológico, con carta dental exigida por el ICBF, se hace una evolución en la historia clínica institucional y una carta dirigida a los padres donde se anotan sugerencias para mejorar la higiene bucal de la paciente y se remite a consulta con especialista para su respectivo tratamiento y mantener las visitas periódicas de control cada 3 meses y se entregó a los cuidadores de la fundación el cepillo dental con el que se realizó la fase de higiene oral.

\section{DISCUSIÓN}

Es de aclarar la importancia de adecuar el entorno para estas intervenciones en salud oral, así en el caso de la atención en el consultorio, se debe permitir un ajuste cuidadoso de la unidad, para 
proporcionar la estabilidad y el soporte necesarios, también se recomienda la atención en la silla de ruedas. Otras recomendaciones son programar la visita del paciente en horario temprano del día y en caso de espasticidad o la no cooperación del paciente, se puede mantener la boca abierta haciendo uso de tacos de mordida; es también fundamental que el odontólogo sea amable, atento y evite movimientos bruscos que puedan desencadenar espasmos musculares o rigidez en el paciente ${ }^{(30)}$, así mismo, los instrumentos afilados deben usarse con extrema precaución para evitar lesiones, igualmente debe tenerse en cuenta siempre que los pacientes con parálisis cerebral a menudo tienen dificultad para enjuagar y escupir adecuadamente De otro lado, la aparatología de ortodoncia o protésicas son recomendables solo si la discapacidad es leve para minimizar el riesgo de fractura y aspiración. En ocasiones ante la poca o total ausencia de cooperación es posible necesitar de sedación o anestesia general, sobre todo en tratamientos invasivos y pacientes con dificultades respiratorias y convulsiones (16).

En cuanto a los hallazgos a nivel oral en la paciente de estudio, se pudo observar que presentaba deficiente higiene oral, lesiones de caries activas en el maxilar inferior, así como enfermedad periodontal, mal oclusión y sialorrea, permitiendo así evidenciar la relación, ya reportada (16), entre la presencia de la agresión neurológica en los niños con parálisis cerebral y el aumento del riesgo y predisposición a desarrollar afecciones y enfermedades bucales.

De otro lado, es importante recordar que la paciente se encuentra bajo los cuidados de la Fundación FE, con la intención de restituir algunos derechos vulnerados por la misma condición sistémica y socioeconómica que ella presenta y se encuentra bajo el cuidado la mayor parte del día del funcionario a cargo en dicha fundación, por lo cual deben analizarse y plantearse un protocolo de 
manejo para el paciente institucionalizado junto con las respectivas sugerencias de cuidado en casa. Estrategias de éxito para atención de dichos pacientes lo que permitan mejorar sus condiciones de salud oral, promover la salud oral y consecuentemente su calidad de vida de esta población vulnerable.

La deficiente higiene oral evidenciada en la paciente soporta la necesidad de promover y aplicar la enseñanza y el entrenamiento, impartido por los estudiantes de Odontología, tanto a padres como a cuidadores de las instituciones reforzando y recordando, tanto a profesores como a padrescuidadores, la importancia de las prácticas saludables bucales, evaluando constantemente el índice de placa bacteriana, ayudando a la mejora de la higiene bucal de los usuarios, valorando y haciendo informes de los pacientes que necesitan urgentemente atención odontológica y previniendo mayores complicaciones orales futuras. Estas labores resaltan el papel fundamental del estudiante de Odontología para tal fin destacando su labor fundamental en las áreas de proyección social y ayuda a la comunidad y permite en ellos desarrollar la iniciativa de generar nuevos conocimientos, su desarrollo integral como futuros profesionales, proactividad, concientización en las necesidades del colectivo y la contribución a las labores sociales con las poblaciones más necesitadas.

Vale la pena recordar que las enfermedades de la cavidad oral, principalmente en pacientes con parálisis cerebral, pueden manifestarse con mayor frecuencia debido a la falta de información por parte de las personas responsables del paciente, a la dificultad del cuidador para abrirles la boca y a la falta de acceso de las personas con dicha discapacidad a servicios odontológicos especializados. Es de resaltar que, al acceder a los servicios de salud, es importante tener en cuenta por parte del personal que presta dicha atención requiere de empatía para establecer vínculos con 
el paciente, la familia y el cuidador ya que de un buen entrenamiento y aceptación en hábitos de salud oral ayudará en el mejor pronóstico de cualquier tratamiento odontológico (30).

De otro lado, en los hallazgos clínicos de la paciente también se observó maloclusión y dependencia de alimentación en forma de licuados (semi-líquida) debido al tipo de parálisis hipotónica que presenta, éste desequilibrio neuromuscular, junto a la falta de una correcta masticación no permite el desarrollo adecuado de la musculatura y frena el correcto desarrollo de ambos maxilares; estos hallazgos permiten resaltar principalmente la importancia que tiene la masticación en función del desarrollo de los músculos periorales y del sistema estomatognático (16).

Adicionalmente una de las comorbilidades que se evidencio en la paciente fue la epilepsia focal, lo cual conlleva al uso de medicamentos anticonvulsivos que pueden generar mayor predisposición al desarrollo de enfermedades gingivales asociadas con la acumulación de biopelícula debida tanto a la dificultad de realizar higiene oral como a la alteración del flujo salival. (30-35). Así entonces, la evidencia de Sialorrea constante observada en al paciente de estudio, podría estar asociada a factores como la disfunción en la coordinación de los mecanismos de deglución, succión y masticación (parálisis pseudo-bulbar) y la apertura de la boca; es de resaltar que este hallazgo de la paciente se reporta en hasta el $30 \%$ de los niños con parálisis cerebral llegando a afectar la nutrición en dicha población $(37,38)$. Debe considerarse además que la presencia de babeo severo puede empeorar con algunos medicamentos antiepilépticos y puede además estar relacionado con una mayor producción de saliva secundaria a una lesión oral irritante como caries dental o infección, esta sialorrea es un aspecto de difícil manejo y contribuye al empeoramiento de la salud dental del paciente; adicionalmente a esta característica en los pacientes con parálisis cerebral se 
puede evidenciar reflujo gastroesofágico (ERGE) el cual puede generar en ellos regurgitación, vómitos y afectar finalmente su salud dental produciendo erosiones dentales (16).

De otro lado, se observó en la paciente un recuento de placa elevado (100 \%) asociado a una deficiente higiene oral, por lo cual se hizo la demostración de una correcta técnica de cepillado y uso de aditamentos de cuidado oral tanto a la profesora de la Fundación como a los estudiantes, resaltando durante la socialización de este proceso la importancia de que el cuidado dental, la higiene en el hogar y el las instituciones de cuidado, deben promoverse desde el principio del ingreso de los pacientes a estas entidades (39). Con niños de más difícil manejo se necesita una restricción más extrema por parte de ambos padres, para lo cual las manos del paciente pueden ser retenidas por una segunda o tercera persona para una limpieza oral efectiva; de otro lado, con el fin de alentar la independencia de los niños con discapacidades motrices más leves, un cepillo de dientes eléctrico puede ser utilizado con eficacia. En general se debe estimular por parte de los estudiantes de Odontología a todos los cuidadores el uso de los implementos de higiene bucal como crema dental con flúor, cepillo, porta sedas, la aplicación de enjuague por medio de gasa e igualmente los cuidadores deben conocer la existencia de abridores de boca. Los anteriores elementos deberán ser usados ya que la salud oral se reconoce cada vez más como una base para el bienestar general, y por ende los cuidadores de pacientes con parálisis cerebral deben considerarse un componente importante del equipo de salud oral y deben adquirir conocimientos y competencias en las prácticas de salud bucal en casa ya que estas pueden afectar significativamente la calidad de vida del niño y controlar los costos de tratamientos odontológicos $(16,40)$. Es importante recordar también que el control químico de la placa se ha recomendado como una alternativa y un complemento para el control mecánico de la placa en estos grupos 
especiales de pacientes siendo el gluconato de clorhexidina una de las mejores opciones en este sentido, ya que en pacientes con parálisis cerebral el fácil uso de esta sustancia a través de una gasa proporciona el control eficaz de la placa, la reducción de los efectos adversos y también la reducción de los problemas de deglución en estos pacientes (41).

En general, se resalta que el seguimiento clínico de los pacientes con parálisis cerebral por parte del odontólogo debe ser un aspecto vital, así como conocer completamente su condición médica y el estado y repercusiones a nivel bucal. Igualmente debe tenerse en cuenta que el uso apropiado de técnicas del manejo del comportamiento basadas en el nivel de comprensión del paciente debe ser continuo y que la atención odontológica ideal para el paciente con parálisis cerebral es la prevención temprana (30). Todo este conocimiento previo y el contacto de los estudiantes de odontología con las diferentes discapacidades que se pueden presentar en los pacientes, permite disminuir futuras barreras en la atención odontológica por la falta de preparación en las diferentes condiciones o por el miedo que constantemente se presenta en algunos profesionales de la salud $(32,33)$.

\section{CONCLUSIONES}

La paciente de estudio evidenció a nivel oral la presencia de caries activas en el maxilar inferior, así como enfermedad periodontal, mal oclusión y sialorrea evidenciando la relación existente entre la presencia de la agresión neurológica en los niños con parálisis cerebral y el aumento del riesgo y predisposición a desarrollar afecciones y enfermedades bucales. 
Así entonces, la enseñanza y el entrenamiento tanto a padres como a cuidadores de las instituciones (donde los niños permanecen la mayor parte del día) por parte de los estudiantes rotantes de cómo debe realizarse una buena higiene bucal, es primordial, por lo cual los estudiantes de Odontología socializaron y estimularon a todos los cuidadores sobre el conocimiento de la existencia de abridores de bocas, así mismo se indicó y ejemplifico el correcto y necesario uso de los implementos de higiene bucal como crema dental con flúor, cepillo, seda o enhebradores, y la aplicación de enjuague por medio de gasa. Cabe aclarar que ésta práctica no cambia el hecho de que la profesora del salón no tenga tiempo para realizar estas actividades de prevención, ya que está encargada de 6 paciente al mismo tiempo, todos dependientes completamente de ella, los estudiantes rotantes solamente van los martes y jueves y al revisar nuevamente a la paciente, se encontró con el mismo recuento alto de placa bacteriana necesitandose acompañamiento diario a los pacientes. Éste hecho nos lleva a pensar y reflexionar en futuros proyectos como lo son la creación de un protocolo institucionalizado del manejo en salud oral para la Fundación junto con la implementación de un plan de actividades diarias para los cuidadores. También los estudiantes rotantes pueden aportar a la comunidad herramientas de ayuda individualizadas.

En general el presente caso de estudio aporta al conocimiento y evidencia de que el paciente diversamente funcional se ve beneficiado por las rotaciones hechas en la Fundación Fe, ya que los estudiantes están continuamente reforzando y recordando, tanto a profesores como a padrescuidadores, la importancia de las prácticas saludables bucales, evaluando constantemente el índice de placa bacteriana, ayudando a la mejora de la higiene bucal de los usuarios, valorando y haciendo informes de los pacientes que necesitan urgentemente atención odontológica y previniendo mayores complicaciones futuras. En cuanto a los beneficios a los estudiantes, ésta práctica crea 
la iniciativa de generar nuevos conocimientos, el desarrollo integral como futuros profesionales, proactividad, concientización en las necesidades del colectivo y la contribución a las labores sociales con las poblaciones más necesitadas.

\section{REFERENCIAS}

1. Organización Mundial de la Salud (OMS). Discapacidad y salud. Ginebra, Suiza: OMS; 16 de enero de 2018. Disponible en: https://www.who.int/es/news-room/fact-sheets/detail/disabilityand-health

2. Fundación Fe. Nosotros. (página en internet). Bogotá, Colombia: la Fundación; 2018. Disponible en: https://www.fundacionfe.org/nosotros.html.

3. Moimaz SAS, Saliba NA, Zina LG, Saliba O, Saliba CAS. Práticas de ensino-aprendizagem com base em cenários reais. Interface (Botucatu). 2010; 14(32): 69-79.

4. Castilho LS, Resende LS, Barros AC, Lacerda DC, Marques EE, Castro N, Pacheco A. Atendimento odontológico a pacientes com necessidades especiais: Considerações a respeito de um projeto de extensão. Rev ELO. 2013 Jul; 2(1): 17-32.

5. Jara AF, Motta E. Orientação e motivação em saúde bucal como aliadas no tratamento odontológico do deficiente mental. Interbio. 2015 Jul-Dez; 9(2): 34-43.

6. Morita MC, Kriger L, Carvalho AC, Haddad AE. Implantação das directrices curriculares nacionales em odontología. Maringá, Paraná, Brasil: Associação Brasileira de Ensino Odontológico, Organização Pan-Americana da Saúde, Ministerio de Saúde; 2007. Disponível em: http://www.abeno.org.br/aadm/images/stories/downloads/implantacao_das_diretrizes_curriculare s_nacionais_abeno_2007.pdf 
7. González Castro GE. Salud bucal individual y colectiva del niño y del adolescente: una experiencia pedagógica en la Universidad Nacional de Colombia. Acta Odontol Colomb. 2017 ene-jun; 7(1): 101-19.

8. Jacomine J, Ferreira R, Campos A, Sant'Ana A, de Rezende ML, Greghi RL, Damantr C, Zangrando M. Saúde bucal e pacientes com necessidades especiais: percepções de graduandos em odontologia da FOB-USP. Rev ABENO. 2018; 18(2): 45-54.

9. Horruitiner Silva P. El reto de la transformación curricular. Rev Iberoam Educ (Organización de Estados Iberoamericanos). 2006 Oct 25; 40(3): 25.

10. Organización Mundial de la Salud (OMS). Clasificación internacional del funcionamiento, de la discapacidad y de la salud (CIF). Madrid, España: OMS; Organización Panamericana de la Salud; Ministerio de Trabajo y Asuntos Sociales, Secretaría General de Asuntos Sociales, Instituto de Migraciones y Servicios Sociales; 2001.

11. República de Colombia. Censo general 2005. Discapacidad. Personas con limitaciones permanentes. Bogotá, Colombia: Departamento Nacional de Estadística; 2005. [consultado: 2019 febrero 7]. Disponible en: https://www.dane.gov.co/files/censos/discapacidad/preva_indices.pdf

12. Ministerio de Salud y Protección Social, República de Colombia. Registro para la localización y caracterización de personas con discapacidad - RLCPD. Bogotá, Colombia: Minsalud; 2019. [consultado: 2019 abril 23]. Disponible https://www.minsalud.gov.co/proteccionsocial/promocion-social/Discapacidad/Paginas/registrolocalizacion.aspx.

13. Ministerio de salud - Protección social. [homepage en Internet, datos compartidos, sala situacional 2018]. Bogotá ;c2019 [última actualización: martes, 23 de abril de 2019] ; consultado el 23 de abril de 2019 
14. Giraldo Zuluaga MC, Martínez Delgado CM, Cardona Gómez N, Gutierrez Pineda JL, Giraldo Moncada KA, Jiménez Ruiz PM. Manejo de la salud bucal en discapacitados. Artículo de revisión. CES Odontología. 2017; 30(2): 23-36

15. Rosenbaum P, Paneth N, Leviton A, Goldstein M, Bax M, Damiano D, Dan J, Jacobsson B. A report: the definition and classification of cerebral palsy April 2006. Dev Med Child Neurol Suppl 2007; 109(109): 8-14.

16. Basil M. Jan, B. M, Jan, M. M. Dental health of children with cerebral palsy. Neurosciences, 2016; 21(4): 314-18.

17. World Health Organization Geneva. International Classification of Functioning, Disability and Health. WHO Library Cataloguing-in-Publication Data. 2001.

18. Wimalasundera N, Stevenson VL. Cerebral palsy. Practical Neurology 2016; 16(3): 184

19. Oskoui M, Coutinho F, Dykeman J, Jetté N, Pringsheim T. An update on the prevalence of cerebral palsy: a systematic review and meta-analysis. Dev Med Child Neurol. 2013 Jun; 55(6): 509-19. doi: 10.1111/dmcn.12080. Epub 2013 Jan 24.Review. Erratum in: Dev Med Child Neurol. 2016 Mar; 58(3): 316.

20. Póo P. Parálisis cerebral infantil. Protocolos Diagnóstico Terapeúticos de la AEP (Asociación Española de Pediatría) Neurología Pediátrica 2008; 271-77.

21. Sanger TD, Delgado MR, Gaebler-Spira D, Hallett M, Mink JW, and the Task Force on. Childhood Motor Disorders. Classification and definition of disorders causing hypertonia in childhood. Pediatrics, 2003; 111: e89-97. doi: 10.1542/peds.111.1.e89

22. Edebol-Tysk K, Hagber B. Epidemiology of spastic tetraplegic cerebral palsy in Sweden: I. Impairments and disabilities. Neuropediatrics. 1989; 20: 41-5. doi:10.1055/s-2008-1071263 
23. Calzada C,Vidal CA, Díaz G. Parálisis cerebral infantil: definición y clasificación a través de la historia. Rev Mex Ortop Ped 2014; 16(1): 6-10.

24. Yeargin-Allsopp M, Van Naarden Braun K et al. Prevalence of cerebral palsy in 8 year old children in three areas of the United States in 2002: a multisite collaboration. Pediatrics. 2008; 121(3): 547-54. doi: 10.1542/peds.2007-1270.

25. Sanger TpD. Movement disorders in cerebral palsy. Journal of Pediatric Neurology. 2015; 13(4): 198-207 doi:http://dx.doi.org.ezproxy.unbosque.edu.co/10.1055/s-0035-1558866

26. Colver A, Fairhurst C, O D Pharoah P. Cerebral palsy. The Lancet April 52014 383: 124049. doi: 10.1016/S0140-6736(13)61835-8

27. Ashwal S, Russman BS, Blasco PA, et al, Quality Standards Subcommittee of the American Academy of Neurology; Practice Committee of the Child Neurology Society. Practice parameter: diagnostic assessment of the child with cerebral palsy: report of the Quality Standards Subcommittee of the American Academy of Neurology and the Practice Committee of the Child Neurology Society. Neurology 2004; 62: 851-63. doi:10.1212/01.WNL.0000117981.35364.1B

28. Wimalasundera N, Stevenson VL. Cerebral palsy Practical Neurology 2016; 16: 184-94. doi:http://dx.doi.org.ezproxy.unbosque.edu.co/10.1136/practneurol-2015-001184

29. Potharaju, N. Seizures in cerebral palsy. Indian Journal of Cerebral Palsy, 2016; 2(1): 3-21 doi:http://dx.doi.org.ezproxy.unbosque.edu.co/10.4103/2395-4264.188150

30. Da Matta, GS, Barriviera M, Marsiglio, AdA, Peruchi, CMdS. \& Miranda, A. F. Preventive and surgical intervention in patient with cerebral palsy - case report. RSBO. 2013; 10(3): 27883. 
31. Abanto, J, Ortega, AO, Raggio, D P, Bonecker M., Mendes FM, Ciamponi, AL. Impact of oral diseases and disorders on oral-health-related quality of life of children with cerebral palsy. Special Care in Dentistry. 2014; 34(2), 56-63. doi:http://dx.doi.org.ezproxy.unbosque.edu.co/10.1111/scd.12028

32. Costa PHM, Costa MABT, Pereira FM. The clinic-epidemiological profile of patients with cerebral palsy treated in a dentistry center in Distrito Federal, Brazil. Com Ciências Saúde. 2007; 18(2): 129-39.Id: lil-484723

33. Dumitru A. study on the barriers and support for participation to social life of children with disabilities. Euromentor Journal 2017; 8(1): 130-48. Retrieved from http://ezproxy.unbosque.edu.co:2048/login?url=https://search-proquestcom.ezproxy.unbosque.edu.co/docview/1907286202?accountid=41311

34. García VV, Bego V, Morales MC. Alteraciones bucodentales prevalentes en una población con parálisis cerebral. Odontol Pediátr. 2016; 24(2): 117-24.

35. Cardoso,AMR., Gomes LN, Silva CRD, Soares RDSC, de Abreu MHNG, Padilha, WWN, Cavalcanti AL. Dental caries and periodontal disease in brazilian children and adolescents with cerebral palsy. International Journal of Environmental Research and Public Health, 2015. 12(1), 335-353. doi:10.3390/ijerph120100335

36. El Ashiry EA, Alaki SM, Nouri SM. Oral Health Quality of Life in Children with Cerebral Palsy: Parental Perceptions. Journal of Clinical Pediatric Dentistry [Internet]. 2016; Jun 6 40(5): 375-87.

37. Diéguez-Pérez, M., de Nova-García, M.-J., Mourelle-Martínez, M. R., \& Bartolomé-Villar, B. Oral health in children with physical (Cerebral Palsy) and intellectual (Down Syndrome) 
disabilities: Systematic review I. Journal of Clinical and Experimental Dentistry. 2016); 8(3): e337-e343. http://doi.org/10.4317/jced.52922

38. Nohelia María Pérez Bejarano, Ferreira Gaona, M. I., Clarisse, V. D., \& Sanabria Vázquez, D. A. Salud bucal en adolescentes con discapacidad del centro de educación especial San Miguel, de Guarambaré, Paraguay. Revista De La Facultad De Odontología Universidad De Antioquia, 2017; 29(1).

39. Guideline on management of dental patients with special health care needs. Pediatric Dentistry [Internet]. [cited 2019 Jun 4]; 38(6): 171-6.

40. Cannon k, Ryan B, Stowe M. Consideration for care for your patient with cerebral palsy. A Literature Review. Virginia Commonwealth University Department of General Practice Dental Hygiene program. 2018.

41. Maiya, A, Shetty, YR, Rai K, Padmanabhan V, Hegde, AM. Use of different oral hygiene strategies in children with cerebral palsy: A comparative study. Journalof International Society of Preventive \& Community Dentistry. 2015; 5(5): 389-93. http://doi.org/10.4103/22310762.165925 